Nonl. Analysis and Differential Equations, Vol. 2, 2014, no. 2, 91 - 104

HIKARI Ltd, www.m-hikari.com

http://dx.doi.org/10.12988/nade.2014.432

\title{
Almost Periodic and Almost Automorphic Solutions to Some Classes of Stochastic Neutral Evolution Equations
}

\author{
Yan Zhao \\ Department of Electronic and Information Engineering \\ Leshan Vocational Technical College \\ Leshan, 614000, P.R. China
}

Copyright (c) 2014 Yan Zhao. This is an open access article distributed under the Creative Commons Attribution License, which permits unrestricted use, distribution, and reproduction in any medium, provided the original work is properly cited.

\begin{abstract}
We investigate some classes of stochastic neutral evolution equations in a real separable Hilbert space. Sufficient conditions for the existence and uniqueness of square-mean almost periodic and square-mean almost automorphic mild solutions are derived with the help of the Banach fixed point theorem. An example is given to illustrate the theory.
\end{abstract}

Keywords: Square-mean almost periodic; Square-mean almost automorphic; fixed point; Exponentially stable semigroup

\section{Introduction}

The concept of an almost periodic function was introduced by Bohr for numerical valued functions and extended by Bochner for functions with values in Polish spaces. In recent years, the existence of almost periodic on different kinds of deterministic differential equations has been 
considerably investigated in lots of publications [1-8] because of its significance and applications in physics, mechanics and mathematical biology.

In [9], Bezandry and Diagana introduced a new concept of a square-mean almost periodicity. They established the existence and uniqueness of square-mean almost periodic mild solutions to some stochastic differential equations and some functional integro-differential stochastic evolution equations in $[9,10]$.

The concept of almost automorphy was first initiated by Bochner in his landmark paper [11]. Since then the theory of almost automorphic functions has found several developments and applications in the theory of abstract differential equations, partial differential equations, functionaldifferential equations, and integro-differential equations. For more on these and related issues, we refer the reader to [12-16] and the references therein.

In a very recent paper [17], Fu and Liu introduced a new concept of a square-mean almost automorphic stochastic process. They established the existence and uniqueness of square-mean almost automorphic mild solutions to some stochastic differential equations.

Neutral differential equations arise in many areas of applied mathematics and for this reason, this type of equation has received much attention in recent years. The existence of almost periodic and almost automorphic solutions to deterministic neutral differential equations has been considerably investigated in lots of publications $[1,4,13]$. However, The existence of square-mean almost periodic and square-mean almost automorphic solutions to stochastic neutral differential equations has not been considered. Motivated by the above discussion, We give in this work some sufficient conditions for the existence and uniqueness of square-mean almost periodic and square-mean almost automorphic mild solutions to some classes of stochastic neutral evolution equations.

\section{Preliminaries}

For details of this section, we refer the reader to Da Prato and Zabczyk [18]. Throughout this paper, $H$ and $K$ will denote real separable Hilbert spaces with respective norms $\|\cdot\|_{H}$ and $\|\cdot\|_{K}$. Let $\left(\Omega, \mathrm{F},\left\{\mathrm{F}_{t}\right\}_{t \geq 0}, P\right)$ be a complete probability space with a filtration $\left\{\mathrm{F}_{t}\right\}_{t \geq 0}$ satisfying the usual conditions (i.e, it is right continuous and $\mathrm{F}_{0}$ contains all $\mathrm{P}$-null sets). We denote by $L_{2}(K ; H)$ the space of all Hilbert-Schmidt operators acting between $K$ and $H$ equipped with the Hilbert-Schmidt norm $\|\cdot\|_{2}$. For a symmetric nonnegative operator $Q \in L_{2}(K ; H)$ with finite trace we assume that $W(t), t \in R$ is a $Q$-Wiener process defined on $\left(\Omega, \mathrm{F},\left\{\mathrm{F}_{t}\right\}_{t \geq 0}, P\right)$ and with values in $K$.

The collection of all strongly measurable, square-integrable $H$-valued random variables, denoted by $L^{2}(P ; H)$, is a Banach space equipped with norm $\|X\|_{L^{2}(P ; H)}=\left(E\|X\|_{H}{ }^{2}\right)^{\frac{1}{2}}$, where the expectation $E$ is defined by $E[g]=\int_{\Omega} g(\omega) d P(\omega)$. 
Let $K_{0}=Q^{\frac{1}{2}} K$ and $L_{2}^{0}=L_{2}\left(K_{0} ; H\right)$ with respect to the norm

$$
\|\Phi\|_{L_{2}^{0}}^{2}=\left\|\Phi Q^{\frac{1}{2}}\right\|_{2}^{2}=\operatorname{Tr}\left(\Phi Q \Phi^{*}\right) .
$$

We are concerned with the existence almost periodic and almost automorphic solutions to the class of stochastic neutral evolution equations of the form

$$
d[x(t)+f(t, B x(t))]=(A x(t)+g(t, C x(t))) d t+\sigma(t, D x(t)) d W(t), \quad t \in R,
$$

where $\mathrm{A}$ is the infinitesimal generator of a uniformly exponentially stable $C_{0}$-semigroup acting on $L^{2}(P ; H), B, C$ and $D$ are densely defined closed linear operators on $L^{2}(P ; H) . f, g$ : $R \times L^{2}(P ; H) \rightarrow L^{2}(P ; H)$ and $\sigma: R \times L^{2}(P ; H) \rightarrow L^{2}\left(P ; L_{2}^{0}\right)$ are jointly continuous functions. One should point out that various stochastic differential equations, stochastic partial differential equations, stochastic integrodifferential equations, and stochastic functional differential equations can be written of the form Eq. (1).

Let $\left(B,\|\cdot\|_{B}\right)$ be a Banach space. Let $C U B\left(R ; L^{2}(P ; B)\right)$ denote the collection of all stochastic processes $X: R \rightarrow L^{2}(P ; B)$, which are continuous and uniformly bounded. It is then easy to check that $C U B\left(R ; L^{2}(P ; B)\right)$ is a Banach space when it is equipped with the norm:

$$
\|X\|_{B, \infty}=\sup _{t \in R}\left(E\|X(t)\|_{B}^{2}\right)^{\frac{1}{2}} .
$$

Let $\left(B_{1},\|\cdot\|_{B_{1}}\right)$ and $\left(B_{2},\|\cdot\|_{B_{2}}\right)$ be Banach spaces. $\mathrm{L}\left(B_{1} ; B_{2}\right)$ and $\mathrm{L}\left(B_{1}\right)$ stand respectively for the class of bounded liner operator from $B_{1}$ to $B_{2}$ and the class of bounded liner operator from $B_{1}$ to itself.

Definition 2.1. A continuous stochastic process $X: R \rightarrow L^{2}(P ; B)$ is said to be square-mean almost periodic if for each $\varepsilon>0$ there exists $l(\varepsilon)>0$ such that any interval of length $l(\varepsilon)$ contains at least a number $\tau$ for which

$$
\sup _{t \in R} E\|X(t+\tau)-X(t)\|_{B}^{2}<\varepsilon
$$

The collection of all stochastic processes $X: R \rightarrow L^{2}(P ; B)$ which are square-mean almost periodic is then denoted by $A P\left(R ; L^{2}(P ; B)\right)$.

The next lemma provides some properties of square-mean almost periodic processes.

Lemma 2.1. [9] If $X$ belongs to $A P\left(R ; L^{2}(P ; B)\right)$, then

(i) the mapping $t \rightarrow E\|X(t)\|^{2}$ is uniformly continuous;

(ii) there exists a constant $N>0$ such that $E\|X(t)\|^{2} \leq N$, for all $t \in R$.

Lemma 2.2. [9] $A P\left(R ; L^{2}(P ; B)\right) \subset C U B\left(R ; L^{2}(P ; B)\right)$ is a closed subspace. 
In view of the above, the space $A P\left(R ; L^{2}(P ; B)\right)$ of square-mean almost periodic processes equipped with the norm $\|\cdot\|_{B, \infty}$ is a Banach space.

Definition 2.2. A function $F: R \times L^{2}\left(P ; B_{1}\right) \rightarrow L^{2}\left(P ; B_{2}\right),(t, Y) \rightarrow F(t, Y)$, which is jointly continuous, is said to be square-mean almost periodic in $t \in R$ uniformly in $Y \in K$ where $K \subset L^{2}\left(P ; B_{1}\right)$ is a compact if for any $\varepsilon>0$, there exists $l(\varepsilon, K)>0$ such that any interval of length $l(\varepsilon, K)$ contains at least a number $\tau$ for which

$$
\sup _{t \in R} E\|F(t+\tau, Y)-F(t, Y)\|_{B_{2}}^{2}<\varepsilon
$$

for each stochastic process $Y: R \rightarrow K$.

Lemma 2.3. [9] Let $F: R \times L^{2}\left(P ; B_{1}\right) \rightarrow L^{2}\left(P ; B_{2}\right),(t, Y) \rightarrow F(t, Y)$ be square-mean almost periodic in $t \in R$ uniformly in $Y \in K$ where $K \subset L^{2}\left(P ; B_{1}\right)$ is a compact. Suppose that $F$ is Lipschitz in the following sense:

$$
E\|F(t, Y)-(t, Z)\|_{B_{2}}^{2} \leq M E\|Y-Z\|_{B_{1}}^{2}
$$

for all $Y, Z \in L^{2}\left(P ; B_{1}\right)$ and for each $t \in R$, where $M>0$. Then for any square-mean almost periodic process $\Phi: R \rightarrow L^{2}\left(P ; B_{1}\right)$, the stochastic process $t \rightarrow F(t, \Phi(t))$ is square-mean almost periodic.

Definition 2.3. A continuous stochastic process $X: R \rightarrow L^{2}(P ; B)$ is said to be square mean almost automorphic if for every sequence of real numbers $\left\{s_{n}^{\prime}\right\}_{n \in N}$ there exist a subsequence $\left\{s_{n}\right\}_{n \in N}$ and a stochastic process $Y: R \rightarrow L^{2}(P ; B)$ such that

$$
\lim _{n \rightarrow \infty} E\left\|X\left(t+s_{n}\right)-Y(t)\right\|_{B}^{2}=0 \quad \text { and } \quad \lim _{n \rightarrow \infty} E\left\|Y\left(t-s_{n}\right)-X(t)\right\|_{B}^{2}=0
$$

hold for each $t \in R$.

The collection of all square-mean almost automorphic stochastic processes $X: R \rightarrow L^{2}(P ; B)$ is denoted by $A A\left(R ; L^{2}(P ; B)\right)$.

Lemma 2.4. [17] $A A\left(R ; L^{2}(P ; B)\right) \subset C U B\left(R ; L^{2}(P ; B)\right)$ equipped with the norm $\|\cdot\|_{B, \infty}$ is a Banach space.

Definition 2.4. A function $F: R \times L^{2}\left(P ; B_{1}\right) \rightarrow L^{2}\left(P ; B_{2}\right),(t, Y) \rightarrow F(t, Y)$, which is jointly continuous, is said to be square-mean almost automorphic in $t \in R$ for each $Y \in L^{2}\left(P ; B_{1}\right)$ if for every sequence of real numbers $\left\{s_{n}^{\prime}\right\}_{n \in N}$ there exist a subsequence $\left\{s_{n}\right\}_{n \in N}$ and a stochastic process $\tilde{F}: R \times L^{2}\left(P ; B_{1}\right) \rightarrow L^{2}\left(P ; B_{2}\right)$ such that

$$
\lim _{n \rightarrow \infty} E\left\|F\left(t+s_{n}, Y\right)-\widetilde{F}(t, Y)\right\|_{B_{2}}^{2}=0 \quad \text { and } \quad \lim _{n \rightarrow \infty} E\left\|\widetilde{F}\left(t-s_{n}, Y\right)-F(t, Y)\right\|_{B_{2}}^{2}=0
$$


Lemma 2.5. [17] Let $F: R \times L^{2}\left(P ; B_{1}\right) \rightarrow L^{2}\left(P ; B_{2}\right),(t, Y) \rightarrow F(t, Y)$ be square-mean almost automorphic in $t \in R$ uniformly in $Y \in L^{2}\left(P ; B_{1}\right)$. Suppose that $F$ is Lipschitz in the following sense:

$$
E\|F(t, Y)-(t, Z)\|_{B_{2}}^{2} \leq M E\|Y-Z\|_{B_{1}}^{2}
$$

for all $Y, Z \in L^{2}\left(P ; B_{1}\right)$ and for each $t \in R$, where $M>0$. Then for any square-mean almost automorphic process $\Phi: R \rightarrow L^{2}\left(P ; B_{1}\right)$, the stochastic process $t \rightarrow F(t, \Phi(t))$ is square mean almost automorphic.

For convenience, we recall from [19] the mild solution to (1) as follows.

Definition 2.5. A stochastic process $x(t):[\delta, \delta+a) \rightarrow L^{2}(P ; H), a>0$, is a mild solution of the neutral stochastic system (1) on $[\delta, \delta+a)$, if the function $s \rightarrow A T(t-s) f(s, x(s-r))$ is intergrable on $[\delta, t)$ for every $\delta<t<\delta+a$, and

$$
\begin{aligned}
x(t)= & T(t)[x(\delta)+f(\delta, B x(\delta))]-f(t, B x(t))-\int_{\delta}^{t} A T(t-s) f(s, B x(s)) d s \\
& +\int_{\delta}^{t} T(t-s) g(s, C x(s)) d s+\int_{\delta}^{t} T(t-s) \sigma(s, D x(s)) d W(s), \quad t \in[\delta, \delta+a)(2)
\end{aligned}
$$

\section{$3 \quad$ Existence results}

Throughout the rest of the work, $\left(G,\|\cdot\|_{G}\right)$ denotes an arbitrary real separable Hilbert spaces, which is continuously embedded into the ground real separable Hilbert spaces $H$.

To prove our results we always assume that the following conditions are satisfied.

(H1) The operator $A$ is the infinitesimal generator of an exponentially stable semigroup $(T(t))_{t \geq 0}$ such that there exist constants $M>0$ and $\delta>0$ with

$$
\|T(t)\|_{L\left(L^{2}(P ; G)\right)} \leq M e^{-\delta t}, \quad \forall t \geq 0 .
$$

Furthermore, the function $s \rightarrow A T(s)$ defined from $(0, \infty)$ into $\mathrm{L}\left(L^{2}(P ; G)\right)$ is strongly (Lebesgue) measurable and there exist a function $\gamma:[0, \infty) \rightarrow[0, \infty)$ and a constant $\omega>0$ with $\rho:=$ $\int_{0}^{\infty} e^{-\omega s} \gamma(s) d s<\infty$ such that

$$
\|A T(s)\|_{\mathrm{L}\left(L^{2}(P ; G)\right)} \leq e^{-\omega s} \gamma(s), \quad s>0 .
$$

$(H 2)$ The functions $f, g(t, x): R \times L^{2}(P ; H) \rightarrow L^{2}(P ; G)$ is a square-mean almost periodic in $t \in R$ uniformly in $x \in \Omega\left(\Omega \subset L^{2}(P ; H)\right.$ being a compact subspace $)$. Moreover, $f, g$ is 
Lipschitz in the following sense: there exist $L_{f}, L_{g}$ for which

$$
\begin{aligned}
& E\|f(t, x)-f(t, y)\|_{G}^{2} \leq L_{f} E\|x-y\|_{H}^{2}, \\
& E\|g(t, x)-g(t, y)\|_{G}^{2} \leq L_{g} E\|x-y\|_{H}^{2},
\end{aligned}
$$

for all stochastic processes $x, y \in L^{2}(P ; H)$ and $t \in R$.

(H3) The functions $\sigma(t, x): R \times L^{2}(P ; H) \rightarrow L^{2}\left(P ; L_{2}\left(K_{0} ; G\right)\right)$ is a square-mean almost periodic in $t \in R$ uniformly in $x \in \Omega\left(\Omega \subset L^{2}(P ; H)\right.$ being a compact subspace ). Moreover, $\sigma$ is Lipschitz in the following sense: there exists $L_{\sigma}$ for which

$$
E\|\sigma(t, x)-\sigma(t, y)\|_{L_{2}\left(K_{0} ; G\right)}^{2} \leq L_{\sigma} E\|x-y\|_{H}^{2}
$$

for all stochastic processes $x, y \in L^{2}(P ; H)$ and $t \in R$.

(H4) $B, C$ and $D$ belong to $\mathrm{L}\left(L^{2}(P ; G) ; L^{2}(P ; H)\right)$ with $\max \left(\|B\|_{\mathrm{L}\left(L^{2}(P ; G) ; L^{2}(P ; H)\right)},\|C\|_{\mathrm{L}\left(L^{2}(P ; G) ; L^{2}(P ; H)\right)}\right.$ $\left.\|D\|_{\mathrm{L}\left(L^{2}(P ; G) ; L^{2}(P ; H)\right)}\right)=h$.

(H5) The functions $f, g(t, x): R \times L^{2}(P ; H) \rightarrow L^{2}(P ; G)$ is a square-mean almost automorphic in $t \in R$ uniformly in $x \in L^{2}(P ; H)$. Moreover, $f, g$ is Lipschitz in the following sense: there exist $L_{f}, L_{g}$ for which

$$
\begin{aligned}
& E\|f(t, x)-f(t, y)\|_{G}^{2} \leq L_{f} E\|x-y\|_{H}^{2}, \\
& E\|g(t, x)-g(t, y)\|_{G}^{2} \leq L_{g} E\|x-y\|_{H}^{2},
\end{aligned}
$$

for all stochastic processes $x, y \in L^{2}(P ; H)$ and $t \in R$.

(H6) The functions $\sigma(t, x): R \times L^{2}(P ; H) \rightarrow L^{2}\left(P ; L_{2}\left(K_{0} ; G\right)\right)$ is a square-mean almost automorphic in $t \in R$ uniformly in $x \in L^{2}(P ; H)$. Moreover, $\sigma$ is Lipschitz in the following sense: there exists $L_{\sigma}$ for which

$$
E\|\sigma(t, x)-\sigma(t, y)\|_{L_{2}\left(K_{0} ; G\right)}^{2} \leq L_{\sigma} E\|x-y\|_{H}^{2}
$$

for all stochastic processes $x, y \in L^{2}(P ; H)$ and $t \in R$.

The proof of our main results require the following technical lemmas.

Lemma 3.1. Suppose that assumptions $(H 1)-(H 2)$ and $(H 4)$ hold. Define the nonlinear operator $\Lambda_{1}$ by: for each $\phi \in A P\left(R ; L^{2}(P ; G)\right)$,

$$
\left(\Lambda_{1} \phi\right)(t):=\int_{-\infty}^{t} A T(t-s) f(s, B \phi(s)) d s
$$

Then $\Lambda_{1}$ maps $A P\left(R ; L^{2}(P ; G)\right)$ into itself. 
Proof. First, let us check that $\Lambda$ is well defined. Indeed, if $\phi \in A P\left(R ; L^{2}(P ; G)\right)$, then $B \phi(s)$ is in $A P\left(R ; L^{2}(P ; G)\right)$ as $B \in \mathrm{L}\left(L^{2}(P ; G) ; L^{2}(P ; H)\right)$. And hence, by Lemma (2.3), the function $f(t, B \phi(t)) \in A P\left(R ; L^{2}(P ; G)\right)$ when $\phi \in A P\left(R ; L^{2}(P ; G)\right)$. In particular, $\|f(t, B \phi(t))\|_{G, \infty}<\infty$. So, we have from the above discussion and $(H 1)$

$$
\begin{aligned}
E\|(\Lambda \phi)(t)\|_{G}^{2} & =E\left\|\int_{-\infty}^{t} A T(t-s) f(s, B \phi(s)) d s\right\|_{G}^{2} \\
& \leq\left(\int_{-\infty}^{t} e^{-\omega(t-s)} \gamma(t-s) d s\right)\left(\int_{-\infty}^{t} e^{-\omega(t-s)} \gamma(t-s) E\|f(s, B \phi(s))\|_{G}^{2} d s\right) \\
& \leq \rho^{2}\|f(t, B \phi(t))\|_{G, \infty}^{2}<\infty .
\end{aligned}
$$

Now, $\Lambda_{1}$ is well defined. By Lemma (2.3), the function $f(t, B \phi(t)) \in A P\left(R ; L^{2}(P ; G)\right)$ when $\phi \in A P\left(R ; L^{2}(P ; G)\right)$. Therefore, for each $\varepsilon>0$ there exists $l(\varepsilon)>0$ such that any interval of length $l(\varepsilon)$ contains at least $\tau$ for which

$$
E\|f(t+\tau, B \phi(t+\tau))-f(t, B \phi(t))\|_{G}^{2}<\frac{\varepsilon}{\rho^{2}}
$$

for each $t \in R$.

We also have that

$$
\begin{aligned}
E\left\|\left(\Lambda_{1} \phi\right)(t+\tau)-\left(\Lambda_{1} \phi\right)(t)\right\|_{G}^{2}= & E\left\|\int_{-\infty}^{t} A T(t-s)[f(s+\tau, B \phi(s+\tau))-f(s, B \phi(s))] d s\right\|_{G}^{2} \\
\leq & \left(\int_{-\infty}^{t} e^{-w(t-s)} \gamma(t-s) d s\right) \\
& \times\left(\int_{-\infty}^{t} e^{-w(t-s)} \gamma(t-s) E\|f(s+\tau, B \phi(s+\tau))-f(s, B \phi(s))\|_{G}^{2} d s\right) \\
\leq & \rho^{2}\|f(s+\tau, B \phi(s+\tau))-f(s, B \phi(s))\|_{G, \infty}^{2} \\
< & \varepsilon
\end{aligned}
$$

In view of the above, $E\left\|\left(\Lambda_{1} \phi\right)(t+\tau)-\left(\Lambda_{1} \phi\right)(t)\right\|_{G}^{2}<\varepsilon$ for each $t \in R$, that is, $\left(\Lambda_{1} \phi\right) t$ is square-mean almost periodic.

Lemma 3.2. Suppose that assumptions $(H 1)-(H 2)$ and $(H 4)$ hold. Define the nonlinear operator $\Lambda_{2}$ by: for each $\phi \in A P\left(R ; L^{2}(P ; G)\right)$,

$$
\left(\Lambda_{2} \phi\right)(t):=\int_{-\infty}^{t} T(t-s) g(s, C \phi(s)) d s
$$

Then $\Lambda_{2}$ maps $A P\left(R ; L^{2}(P ; G)\right)$ into itself.

Proof. The proof is similar to that of Lemma (3.1) with appropriate adaptations. So we omit it. 
Lemma 3.3. Suppose that assumptions $(H 1),(H 3)$ and $(H 4)$ hold. Define the nonlinear operator $\Lambda_{3}$ by: for each $\phi \in A P\left(R ; L^{2}(P ; G)\right)$,

$$
\left(\Lambda_{3} \phi\right)(t):=\int_{-\infty}^{t} T(t-s) \sigma(s, D x(s)) d W(s) .
$$

Then $\Lambda_{3}$ maps $A P\left(R ; L^{2}(P ; G)\right)$ into itself.

Proof. The proof is similar to that of Theorem 3.2 in [9] with appropriate adaptations. So we omit it.

Lemma 3.4. Suppose that assumptions $(H 4)-(H 5)$ hold. Define the nonlinear operator $\Lambda_{4}$ by: for each $\phi \in A A\left(R ; L^{2}(P ; G)\right)$,

$$
\left(\Lambda_{4} \phi\right)(t):=\int_{-\infty}^{t} A T(t-s) f(s, B \phi(s)) d s .
$$

Then $\Lambda_{4}$ maps $A A\left(R ; L^{2}(P ; G)\right)$ into itself.

Proof. First, let us check that $\Lambda_{4}$ is well defined. Indeed, if $\phi \in A A\left(R ; L^{2}(P ; G)\right)$, then $B \phi(s)$ is in $A A\left(R ; L^{2}(P ; G)\right)$ as $B \in \mathrm{L}\left(L^{2}(P ; G) ; L^{2}(P ; H)\right)$. And hence, by Lemma (2.5), the function $f(t, B \phi(t)) \in A A\left(R ; L^{2}(P ; G)\right)$ when $\phi \in A A\left(R ; L^{2}(P ; G)\right)$. In particular, $\|f(t, B \phi(t))\|_{G, \infty}<\infty$. So, we have from the above discussion and $(H 1)$

$$
\begin{aligned}
E\|(\Lambda \phi)(t)\|_{G}^{2} & =E\left\|\int_{-\infty}^{t} A T(t-s) f(s, B \phi(s)) d s\right\|_{G}^{2} \\
& \leq\left(\int_{-\infty}^{t} e^{-\omega(t-s)} \gamma(t-s) d s\right)\left(\int_{-\infty}^{t} e^{-\omega(t-s)} \gamma(t-s) E\|f(s, B \phi(s))\|_{G}^{2} d s\right) \\
& \leq \rho^{2}\|f(t, B \phi(t))\|_{G, \infty}^{2}<\infty .
\end{aligned}
$$

Now, $\Lambda_{4}$ is well defined. To prove that $\left(\Lambda_{4} \phi\right) \in A A\left(R ; L^{2}(P ; G)\right)$, one has to prove that

$$
t \rightarrow \Lambda_{4} \phi(t)=\lim _{r \rightarrow-\infty} \int_{r}^{t} A T(t-s) \varphi(s) d s
$$

is square-mean almost automorphic, where $\varphi(s)=f(s, B x(s))$ for each $s \in R$.

Now let $s_{n}^{\prime}$ be an arbitrary sequence of real numbers, Since $\varphi$ is square-mean almost automorphic, there exists a subsequence $\left(s_{n}\right)$ of $s_{n}^{\prime}$ such that $\lim _{n \rightarrow \infty} E\left\|\varphi\left(t+s_{n}\right)-h(t)\right\|_{G}^{2}=0$ for each $t \in R$ and $\lim _{n \rightarrow \infty} E\left\|\varphi(t)-h\left(t-s_{n}\right)\right\|_{G}^{2}=0$ for each $t \in R$.

Now

$$
\begin{aligned}
& E\left\|\int_{-\infty}^{t+s_{n}} A T\left(t+s_{n}-s\right) \varphi(s) d s-\int_{-\infty}^{t} A T(t-s) h(s) d s\right\|^{2} \\
& \quad=E\left\|\int_{-\infty}^{t} A T(t-s) \varphi\left(s+s_{n}\right) d s-\int_{-\infty}^{t} A T(t-s) h(s) d s\right\|^{2} \\
& \quad \leq E\left\|\int_{-\infty}^{t} A T(t-s)\left[\varphi\left(s+s_{n}\right)-h(s)\right] d s\right\|^{2} \\
& \quad \leq \rho \int_{-\infty}^{t} e^{-\omega(t-s)} \gamma(t-s) E\left\|\varphi\left(s+s_{n}\right)-h(s)\right\|^{2} d s .
\end{aligned}
$$


So, by Lebesgues dominated convergence theorem, $E\left\|\int_{-\infty}^{t+s_{n}} A T\left(t+s_{n}-s\right) \varphi(s) d s-\int_{-\infty}^{t} A T(t-s) \varphi(s) d s\right\|^{2}=$ 0 as $n \rightarrow \infty$ for each $t \in R$.

Similarly, it can be shown that $E\left\|\int_{-\infty}^{t} A T(t-s) \varphi(s) d s-\int_{-\infty}^{t-s_{n}} A T\left(t-s_{n} t-s\right) h(s) d s\right\|^{2}=$ 0 as $n \rightarrow \infty$ for each $t \in R$.

This shows that $\Lambda_{4} \phi(t)$ is a square-mean almost automorphic function.

Lemma 3.5. Suppose that assumptions $(H 1)$ and $(H 4)-(H 5)$ hold. Define the nonlinear operator $\Lambda_{5}$ by: for each $\phi \in A A\left(R ; L^{2}(P ; G)\right)$,

$$
(\Lambda 5 \phi)(t):=\int_{-\infty}^{t} T(t-s) g(s, C \phi(s)) d s
$$

Then $\Lambda_{5}$ maps $A A\left(R ; L^{2}(P ; G)\right)$ into itself.

Proof. The proof is similar to that of Lemma 3.4 with appropriate adaptations. So we omit it.

Lemma 3.6. Suppose that assumptions $(H 1),(H 4)$ and $(H 6)$ hold. Define the nonlinear operator $\Lambda_{6}$ by: for each $\phi \in A A\left(R ; L^{2}(P ; G)\right)$,

$$
\left(\Lambda_{6} \phi\right)(t):=\int_{-\infty}^{t} T(t-s) \sigma(s, D x(s)) d W(s) .
$$

Then $\Lambda_{6}$ maps $A A\left(R ; L^{2}(P ; G)\right)$ into itself.

Proof. The proof is similar to that of Theorem 3.1 in [20] with appropriate adaptations. So we omit it.

Theorem 3.1. Assume the conditions $(H 1)-(H 4)$ hold. Then (1) has a unique square-mean almost period mild solution on $R$ provided that

$$
4\left[h^{2} L_{f}+\rho^{2} R^{2} L_{f}+h^{2} \frac{1}{\delta^{2}} M^{2} L_{g}+h^{2} \frac{1}{\delta^{2}} M^{2} L_{\sigma}\right]<1 .
$$

Proof. Let $\Gamma: A P\left(R ; L^{2}(P ; G)\right) \rightarrow A P\left(R ; L^{2}(P ; G)\right)$ be the map defined by

$$
\begin{aligned}
\Gamma x(t)= & -f(t, B x(t))-\int_{-\infty}^{t} A T(t-s) f(s, B x(s)) d s \\
& +\int_{-\infty}^{t} T(t-s) g(s, C x(s)) d s+\int_{-\infty}^{t} T(t-s) \sigma(s, D x(s)) d W(s), \quad t \in R .(4)
\end{aligned}
$$

From Lemma 2.3, Lemma 3.1, Lemma 3.2 and Lemma 3.3, it follows that the nonlinear operator $\Gamma$ is well defined, that is, it maps $A P\left(R ; L^{2}(P ; G)\right) \rightarrow A P\left(R ; L^{2}(P ; G)\right)$. In the following, we prove that $\Gamma$ is a contraction. 
Clearly,

$$
\begin{aligned}
\|(\Gamma x)(t)-(\Gamma y)(t)\|_{G} \leq & \|f(t, B x(t))-f(t, B y(t))\|_{G}+\left\|\int_{-\infty}^{t} A T(t-s)[f(s, B x(s))-f(s, B y(s))] d s\right\|_{G} \\
& +\left\|\int_{-\infty}^{t} T(t-s)[g(s, C x(s))-g(s, C y(s))] d s\right\|_{G} \\
& +\left\|\int_{-\infty}^{t} T(t-s)[\sigma(s, D x(s))-\sigma(s, D y(s))] d W(s)\right\|_{G} .
\end{aligned}
$$

Since $(a+b+c+d)^{2} \leq 4 a^{2}+4 b^{2}+4 c^{2}+4 d^{2}$, we have

$$
\begin{aligned}
E\|(\Gamma x)(t)-(\Gamma y)(t)\|_{G}^{2} \leq & 4 E\|f(t, B x(t))-f(t, B y(t))\|_{G}^{2} \\
& +4 E\left\|\int_{-\infty}^{t} A T(t-s)[f(s, B x(s))-f(s, B y(s))] d s\right\|_{G}^{2} \\
& +4 E\left\|\int_{-\infty}^{t} T(t-s)[g(s, C x(s))-g(s, C y(s))] d s\right\|_{G}^{2} \\
& +4 E\left\|\int_{-\infty}^{t} T(t-s)[\sigma(s, D x(s))-\sigma(s, D y(s))] d W(s)\right\|_{G}^{2} .
\end{aligned}
$$

We first evaluate the first term of the right-hand side as follows:

$$
E\|f(t, B x(t))-f(t, B y(t))\|^{2} \leq h^{2} L_{f}\|x-y\|_{G, \infty}^{2} .
$$

As to the second term, it follows from $(H 1)$ that

$$
\begin{aligned}
& E\left\|\int_{-\infty}^{t} A T(t-s)[f(s, B x(s))-f(s, B y(s))] d s\right\|^{2} \\
& \leq E\left[\int_{-\infty}^{t} e^{-\omega(t-s)} \gamma(t-s)\|f(s, B x(s))-f(s, B y(s))\| d s\right]^{2} \\
& \leq\left(\int_{-\infty}^{t} e^{-\omega(t-s)} \gamma(t-s) d s\right)^{2} \sup _{t \in R} E\|f(s, B x(s))-f(s, B y(s))\|^{2} \\
& \leq \rho^{2} h^{2} L_{f}\|x-y\|_{G, \infty}^{2} .
\end{aligned}
$$

As to the third term, we obtain that

$$
\begin{aligned}
& E\left\|\int_{-\infty}^{t} T(t-s)[g(s, C x(s))-g(s, C y(s))] d s\right\|^{2} \\
& \leq M^{2} E\left\|\int_{-\infty}^{t} e^{-\delta(t-s)}[g(s, C x(s))-g(s, C y(s))] d s\right\|^{2} \\
& \leq h^{2} \frac{1}{\delta^{2}} M^{2} L_{g}\|x-y\|_{G, \infty}^{2} .
\end{aligned}
$$

As far as the last term is concerned, we use an estimate on the Itô integral established in [21, Proposition 1.9] and obtain:

$$
\begin{aligned}
& E\left\|\int_{-\infty}^{t} T(t-s)[\sigma(s, D x(s))-\sigma(s, D y(s))] d W(s)\right\|^{2} \\
& \leq M^{2} E\left(\int_{-\infty}^{t} e^{-\delta(t-s)} d s\right) \int_{-\infty}^{t} e^{-\delta(t-s)}\|\sigma(s, D x(s))-\sigma(s, D y(s))\|^{2} d s \\
& \leq h^{2} \frac{1}{\delta^{2}} M^{2} L_{\sigma}\|x-y\|_{G, \infty}^{2} .
\end{aligned}
$$


Thus, by combining, it follows that

$$
\|\Gamma x-\Gamma y\|_{G, \infty} \leq 4\left[h^{2} L_{f}+\rho^{2} h^{2} L_{f}+h^{2} \frac{1}{\delta^{2}} M^{2} L_{g}+h^{2} \frac{1}{\delta^{2}} M^{2} L_{\sigma}\right]\|x-y\|_{G, \infty} .
$$

which shows that $\Gamma(\cdot)$ is a contraction and completes the proof of this result.

When $f=0, B=C=D=I$, system (1) reduces to the following stochastic system

$$
d x(t)=(A x(t)+g(t, x(t))) d t+\sigma(t, x(t)) d W(t), \quad t \in R,
$$

Corollary 1. Assume the conditions $(H 1)-(H 3)$ hold. Then (5) has a unique square-mean almost period mild solution on $R$ provided that

$$
2\left[\frac{1}{\delta^{2}} M^{2} L_{g}+\frac{1}{\delta^{2}} M^{2} L_{\sigma}\right]<1 .
$$

Proof. The proof is similar the proof of Theorem 3.1, so we omit it here.

Remark 3.1. Corollary 1 in the case $G=H$ is the main result of [9].

Theorem 3.2. Assume the conditions $(H 1)$ and $(H 4)-(H 6)$ hold. Then (1) has a unique square-mean almost automorphic mild solution on $R$ provided that

$$
4\left[h^{2} L_{f}+\rho^{2} h^{2} L_{f}+h^{2} \frac{1}{\delta^{2}} M^{2} L_{g}+h^{2} \frac{1}{\delta^{2}} M^{2} L_{\sigma}\right]<1 .
$$

Proof. Let $\Gamma: A A\left(R ; L^{2}(P ; G)\right) \rightarrow A A\left(R ; L^{2}(P ; G)\right)$ be the map defined by

$$
\begin{aligned}
\Gamma x(t)= & -f(t, x(t-r))-\int_{-\infty}^{t} A T(t-s) f(s, x(s-r)) d s \\
& \left.+\int_{-\infty}^{t} T(t-s) g(s, x(s-r)) d s+\int_{-\infty}^{t} T(t-s) \sigma(s, x(s-r)) d W(s), \quad t \in \mathbb{8} .\right)
\end{aligned}
$$

From Lemma 2.5, Lemma 3.4, Lemma 3.5 and Lemma 3.6, it follows that the nonlinear operator $\Gamma$ is well defined, that is, it maps $A A\left(R ; L^{2}(P ; G)\right) \rightarrow A A\left(R ; L^{2}(P ; G)\right)$. As the discussion in Theorem 3.1, we can prove that $\Gamma$ is a contraction. So, the proof is completed.

Corollary 2. Assume the conditions $(H 1),(H 5)$ and $(H 6)$ hold. Then $(5)$ has a unique squaremean automorphic mild solution on $R$ provided that

$$
2\left[\frac{1}{\delta^{2}} M^{2} L_{g}+\frac{1}{\delta^{2}} M^{2} L_{\sigma}\right]<1 .
$$

Proof. The proof is similar the proof of Theorem (3.1), so we omit it here.

Remark 3.2. Corollary 2 in the case $G=H$ is the main result of [17]. 


\section{Example}

In this section an example is presented to illustrate Theorem 3.1. We consider the following neutral stochastic equation.

$$
\begin{gathered}
\frac{\partial}{\partial t} x(t, \xi)=\frac{\partial^{2}}{\partial \xi^{2}} x(t, \xi)-\frac{\partial}{\partial t} f(t, B x(t, \xi))+g(t, C x(t, \xi))+\sigma(t, D x(t, \xi)) d W(t) \\
0 \leq \xi \leq 1 .
\end{gathered}
$$

with the Dirichlet boundary conditions

$$
x(t, 0)=x(t, 1)=0 .
$$

Following a well-known approach of [22] we will rewrite system (10)) and (11) as an evolution equation

$$
d[x(t)+f(t, B x(t))]=(A x(t)+g(t, C x(t))) d t+\sigma(t, D x(t)) d W(t)
$$

in the space $H=L^{2}[0,1], W(t)$ stands for a standard cylindrical Wiener process in $H$ defined in a stochastic basis $(\Omega, \mathrm{F}, P)$. Let $A: H \rightarrow H$ be the linear operator defined by $A=\left(\partial^{2} / \partial \xi^{2}\right)$, where $D(A)=\left\{y \in H: y, d y / d x\right.$ are absolutely continuous, and $\left.\left(d^{2} / d x^{2}\right) y \in H, y(0)=y(1)=0\right\}$.

Then A generates a symmetric $C_{0}$-semigroup $e^{-t A}$ in $H$ and so that there exists an $M>0$ and $\delta>0$ such that $\left\|e^{-t A}\right\| \leq M e^{-\delta t}$. Also note that there exists a complete orthonormal set $\left\{\zeta_{n}\right\}(n=1,2,3, \ldots)$ of eigenvectors of $A$ with $\zeta_{n}(x)=\sqrt{2 / \pi} \sin n x$. Then, the operator $A^{-\frac{1}{2}}$ is

given by $A^{-\frac{1}{2}} \zeta=\sum_{n=1}^{\infty} n\left\langle\zeta, \zeta_{n}\right\rangle \zeta_{n}$ on the space $D\left[A^{\frac{1}{2}}\right]=\left\{\zeta(\cdot) \in H: \sum_{n=1}^{\infty} n\left\langle\zeta, \zeta_{n}\right\rangle \zeta_{n} \in H\right\}$ (for more details see $[22])$.

For the sake of simplicity, we consider the case where $G=H=L^{2}[0,1]$ and $B=C=D$ are the bounded multiplication operator defined by: $B x=C x=D x=q(x)$ for each $u \in L^{2}[0,1]$ where $q \in L^{\infty}[0,1]$. In this even, $\|B\|_{\mathrm{L}\left(L^{2}(P ; H)\right)}=\|C\|_{\mathrm{L}\left(L^{2}(P ; H)\right)}=\|D\|_{\mathrm{L}\left(L^{2}(P ; H)\right)}=\|q\|_{\infty}$.

Thus, under assumptions $(H 1)-(H 4)$, once (3) holds, an application of Theorem 3.1 yields that (1) has a unique mild solution, which is obviously square-mean almost periodic.

Thus, under assumptions $(H 1)$ and $(H 4)-(H 6)$, once $(7)$ holds, an application of Theorem 3.2 yields that (1) has a unique mild solution, which is obviously square-mean almost automorphic.

\section{References}

[1] E. Hernández M., M.L. Pelicer, Asymptotically almost periodic and almost periodic solutions for partial neutral differential equations, Appl. Math. Lett. 18 (2005) 1265-1272. 
[2] H.R. Henríquez, C.H. Vásquez, Almost periodic solutions of abstract retarded functionaldifferential equations with unbounded delay, Acta Appl. Math. 57 (2) (1999) 105-132.

[3] T. Furumochi, T. Naito, N.V. Minh, Boundedness and almost periodicity of solutions of partial functional differential equations, J. Differential Equations 180 (1) (2002) 125-152.

[4] N.M. Man, N.V. Minh, On the existence of quasi periodic and almost periodic solutions of neutral functional differential equations, Commun. Pure Appl. Anal. 3 (2) (2004) 291-300.

[5] E. Hernández M., M.L. Pelicer, J.P.C. Dos Santos, Asymptotically almost periodic and almost periodic solutions for a class of evolution equations, Electron. J. Differential Equations (61) (2004) 15.

[6] R. Yuan, Existence of almost periodic solutions of neutral functional-differential equations via Liapunov-Razumikhin function, Z. Angew. Math. Phys. 49 (1) (1998) 113-136.

[7] Z.H. Zhao, Y.K. Chang, W.S. Li, Asymptotically almost periodic, almost periodic and pseudo almost periodic mild solutions for neutral differential equations, Nonlinear Anal. RWA 11 (2010) 3037-3044.

[8] H.S. Ding, J. Liang, G.M. N'Guérékata, T.J. Xiao, Pseudo almost periodicity of some nonautonomous evolution equations with delay, Nonlinear Anal. TMA 67 (2007) 1412-1418.

[9] P. Bezandry, T. Diagana, Existence of almost periodic solutions to some stochastic differential equations, Appl. Anal. 117 (2007) 1-10.

[10] P. Bezandry, Existence of almost periodic solutions to some functional integro-differential stochastic evolution equations, Stat. Probabil. Lett. 78 (2008) 2844-2849.

[11] S. Bochner, A new approach to almost periodicity, Proc. Natl. Acad. Sci. USA 48 (1962) $2039 \mathrm{C} 2043$.

[12] Z.H. Zhao, Y.K. Chang, J.J. Nieto, Almost automorphic and pseudo almost automorphic mild solutions to an abstract differential equation in Banach spaces, Nonlinear Anal. TMA 72 (2010) 1886-1894.

[13] T. Diagana, G.M. N'Guérékata, Almost automorphic solutions to some classes of partial evolution equations, Appl. Math. Lett. 20 (2007) 462-466.

[14] T. Diagana, G.M. N'Guérékata, N.V. Minh, Almost automorphic solutions of evolution equations, Proc. Amer. Math. Soc. 132 (11) (2004) 3289-3298.

[15] J.A. Goldstein, G.M. N'Guérékata, Almost automorphic solutions of semilinear evolution equations, Proc. Amer. Math. Soc. 133 (8) (2005) 2401-2408. 
[16] G.M. N'Guérékata, Existence and uniqueness of almost automorphic mild solutions to some semilinear abstract differential equations, Semigroup Forum 69 (2004) 80-86.

[17] M.M. Fu, Z.X. Liu, Square-mean almost automorphic solutions for some stochastic differential equations, Proc. Amer. Math. Soc. (in press), arXiv:1001.3049v1 [math.DS].

[18] G. Da Prato, J. Zabczyk, Stochastic Equations in Infinite Dimensions, Cambridge Univ. Press, Cambridge, 1992.

[19] K. Liu, Stability of Infinite Dimensional Stochastic Differential Equations with Applications, Chapman and Hall, CRC, London, 2004.

[20] Y.K. Chang, et al., Square-mean almost automorphic mild solutions to non-autonomous stochastic differential equations in Hilbert spaces, Computers and Mathematics with Applications (2010), doi:10.1016/j.camwa.2010.11.014.

[21] Ichikawa, A., 1982. Stability of semilinear stochastic evolution equations. J. Math. Anal. Appl. 90 (1), 12-44.

[22] A. Pazy, Semigroups of linear operators and applications to partial differential equations, in: Applied Methematical Sciences, vol. 44, Springer Verlag, New York, 1983.

Received: March 5, 2014 